\title{
LAP2a preserves genome integrity through assisting RPA deposition on damaged chromatin
}

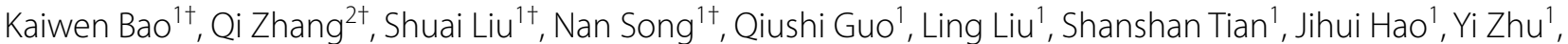 \\ Kai Zhang ${ }^{1}$, Ding $\mathrm{Ai}^{1}$, Jie Yang ${ }^{1}$, Zhi Yao ${ }^{1}$, Roland Foisner ${ }^{3}$ and Lei Shi ${ }^{1,4^{*}}$ (D)
}

*Correspondence:

shilei@tmu.edu.cn

†Kaiwen Bao, Qi Zhang,

Shuai Liu and Nan Song

contributed equally to this

work.

${ }^{4}$ Department

of Biochemistry

and Molecular Biology,

Tianjin Medical University,

22 Qixiangtai Road,

Tianjin 300070, China

Full list of author information

is available at the end of the

article

\begin{abstract}
Background: Single-stranded DNA (ssDNA) coated with replication protein A (RPA) acts as a key platform for the recruitment and exchange of genome maintenance factors in DNA damage response. Yet, how the formation of the ssDNA-RPA intermediate is regulated remains elusive.
\end{abstract}

Results: Here, we report that the lamin-associated protein LAP2a is physically associated with RPA, and LAP2a preferentially facilitates RPA deposition on damaged chromatin via physical contacts between LAP2a and RPA1. Importantly, LAP2a-promoted RPA binding to ssDNA plays a critical role in protection of replication forks, activation of ATR, and repair of damaged DNA. We further demonstrate that the preference of LAP2apromoted RPA loading on damaged chromatin depends on poly ADP-ribose polymerase PARP1, but not poly(ADP-ribosyl)ation.

Conclusions: Our study provides mechanistic insight into RPA deposition in response to DNA damage and reveals a genome protection role of LAP2a.

Keywords: RPA loading, Genome stability, ATR activation, Homologous recombination, LAP2a, PARP1

\section{Background}

During each cell cycle of eukaryotic cells, genetic inheritance is constantly challenged by endogenous or exogenous assaults that can undermine the integrity of the replicating genome [1-3]. In physiological settings, mild forms of replication stress stochastically occur during cell-cycle progression, while the severe forms could be further induced in pathological states such as oncogenic transformation and chemotherapeutic agents, which could lead to DNA damage and instigate genomic instability [4-6]. There are numerous impediments that block the progression of DNA replication forks, including DNA lesions, the transcription machinery, DNA-protein complex, RNA-DNA hybrids, and secondary DNA structures [7-10]. If replication stress persists, stalled forks could

(c) The Author(s) 2022 Open Access This article is licensed under a Creative Commons Attribution 4.0 International License, which permits use, sharing, adaptation, distribution and reproduction in any medium or format, as long as you give appropriate credit to the original author(s) and the source, provide a link to the Creative Commons licence, and indicate if changes were made. The images or other third party material in this article are included in the article's Creative Commons licence, unless indicated otherwise in a credit line to the material. If material is not included in the article's Creative Commons licence and your intended use is not permitted by statutory regulation or exceeds the permitted use, you will need to obtain permission directly from the copyright holder. To view a copy of this licence, visit http:// creativecommons.org/licenses/by/4.0/. The Creative Commons Public Domain Dedication waiver (http://creativecommons.org/publi cdomain/zero/1.0/) applies to the data made available in this article, unless otherwise stated in a credit line to the data. 
be converted into collapsed forks and even DNA double-strand breaks (DSBs) that pose the most serious threat to genome integrity [10]. Although these complexed structures may differ greatly, they all result in the formation of long stretches of single-stranded DNA (ssDNA) that are coated by replication protein A (RPA), a common intermediate product, which, in turn, provides a platform for the recruitment and exchange of genome maintenance factors to respond to stresses or damages and orchestrates a genome surveillance pathway [11-13].

As one of the early phase responders in the replication stress, ssDNA-RPA is the key structure that triggers the activation of serine/threonine kinase Ataxia telangiectasia and Rad3 related (ATR), a master regulator of the DNA damage response (DDR) [3, 11]. Once activated, ATR coordinates with its downstream effectors to counteract adverse effects of replication stress and repair of damaged DNA both by delaying cell-cycle progression and by stabilizing stalled forks $[14,15]$. In support of the notion that RPA-ATR signalling axis is essential for the maintenance of genome integrity, disruption of RPA in mice results in defective repair of DSBs, chromosomal instability, and tumorigenesis [16, 17], and defects of ATR signalling leads to chromosome fragmentation, developmental failures, accelerated aging, and predisposition of carcinogenesis [18-21]. When DSBs occur, RPA sequesters the ssDNA of the resected DSB, allowing the formation of the RAD51-ssDNA nucleoprotein filament to catalyze strand invasion for accurate repair of DSBs by homologous recombination (HR) [22]. These evidences pinpoint the importance of RPA-dependent surveillance network in genome stability.

RPA is composed of heterotrimeric subunits RPA1, RPA2, and RPA3, and it binds to ssDNA with sub-nanomolar affinity and protects it against nucleolytic degradation or breakage as well as removes secondary structures [13,23]. As a stable, flexible, and modular protein complex, RPA contains six oligosaccharide/oligonucleotide binding (OB) folds that are responsible for the association of RPA with a short or long stretch of ssDNA [13, 23]. The largest subunit, RPA1, possesses four OB domains commonly referred to as DNA-binding domains (DBDs): DBD-A, DBD-B, DBD-C, and DBD-F, which are connected by flexible linkers [24-26]. RPA2 has a single OB-fold (DBD-D) to constitute the trimerisation core (Tri-C) with DBD-C of RPA1 and DBD-E of RPA3 [24, 25]. Structural, biochemical, and biophysical studies have shown that each individual DBD from A to E possesses intrinsic ssDNA binding activity, albeit with different affinity $[24,25,27,28]$, and the combinatorial action of these domains enables RPA binding to ssDNA with a defined polarity from $5^{\prime}$ to $3^{\prime}$ using two different modes of binding [24, 25, 27, 29]: the 8 to 10 nucleotides (nt) occluded only by its DBD-A and BDD-B uses an extended conformation with lower affinity, whereas the 28- to 30-nt binding by fully engaged RPA goes along with a compact protein structure and higher affinity. These two different binding modalities appear to act sequentially to facilitate the association of RPA with ssDNA.

A large number of RPA or ssDNA-RPA interaction proteins or protein complexes have been characterized, and ssDNA-RPA complex is believed to act as a key platform to coordinate the arrival and departure of these factors whose combined activities permit the protection of eukaryotic genomes against damages or stresses [13]. Interestingly, several studies have reported that RPA binding to ssDNA could be regulated by defined factors such as histone methyltransferase G9a [30], DNA helicase Fanconi anemia 
group J protein (FANCJ) [31], and tumor suppressor protein phosphatase and tensin homolog (PTEN) [17]. Relevantly, cell division cycle protein 45 (CDC45), a component of the active replicative helicase CDC45/MCM2-7/GINS (CMG), has been proposed to directly load RPA on the emerging nascent ssDNA at replication fork [32], and protein regulator of Ty1 transposition 105 (Rtt105) from yeast has been shown to function as a canonical RPA chaperone that helps deposit RPA at stalled replication forks [33]. These two studies point that RPA could be actively loaded onto ssDNA by chaperone or chaperone-like proteins. Yet, it is still not fully understood, in mammalian cells, how the formation of ssDNA-RPA intermediate generated during replication stress or at DNA lesions is regulated.

Lamin-associated polypeptide 2 alpha (LAP2 $\alpha$ ) is the largest splicing product of the LAP2 gene (TMPO) that encodes six variants sharing a constant $\mathrm{N}$-terminal LAP2, emerin, and MAN1 (LEM) like domain linked through a flexible tether to the LEM domain [34, 35]. Unlike other LAP2 isoforms that are preferentially anchored to the nuclear inner membrane through a transmembrane domain and binding to lamins, the LAP2 $\alpha$ isoform replaces the transmembrane domain with a unique coiled-coil domain in the C-terminal region and localizes throughout the nucleus [34]. This distinct C-terminal region interacts with A-type lamins and defines Lamin $\mathrm{A} / \mathrm{C}$ localization in the nucleoplasm [36], which in turn affects retinoblastoma protein $(\mathrm{pRb})$-mediated regulation of gene expression, and progenitor cell proliferation and differentiation in highly regenerative tissues [37]. However, the activity of LAP2 $\alpha$ in other fundamental cellular processes including DDR and genome stability, dysregulation of which will contribute to tumorigenesis, has not been well documented.

Here, we report that LAP2 $\alpha$ functions to assist RPA deposition and maintain genome stability in mammalian cells. Specifically, we revealed that LAP2 $\alpha$ preserves genome integrity through controlling the formation of ssDNA-RPA complex on damaged chromatin, and LAP2 $\alpha$-promoted RPA loading is required for fork stability, ATR activation, and HR repair of DSBs.

\section{Results}

\section{RPA is physically associated with LAP2a}

To identify potential regulators that control RPA loading onto ssDNA, we employed immunopurification and mass spectrometry with stable isotope labelling with amino acids in cell culture (SILAC) to interrogate free RPA interactome. Quantitative mass spectrometry analysis of FLAG-RPA1 containing protein complex in the presence of DNase revealed that RPA1 was associated with a number of proteins, including RPA2 and RPA3 (Fig. 1A, B, and Additional file 1: Table S1). Interestingly, LAP2 $\alpha$, a previously characterized Lamin $\mathrm{A} / \mathrm{C}$ and $\mathrm{pRB}$-associated nuclear protein, was identified as one of the top candidates (Fig. 1A, B, and Additional file 1: Table S1). Since the enrichment of LAP2 $\alpha$ was almost comparable to that of each RPA subunit (Fig. 1A, B, and Additional file 1: Table S1), we focused on further characterization of LAP2 $\alpha$ in RPA binding and regulation. To confirm the association of LAP $2 \alpha$ with RPA, co-immunoprecipitation experiments were then performed and the results showed that LAP2 $\alpha$ was efficiently coimmunoprecipitated with RPA, and vice versa under DNase treatment (Fig. 1C,D). 


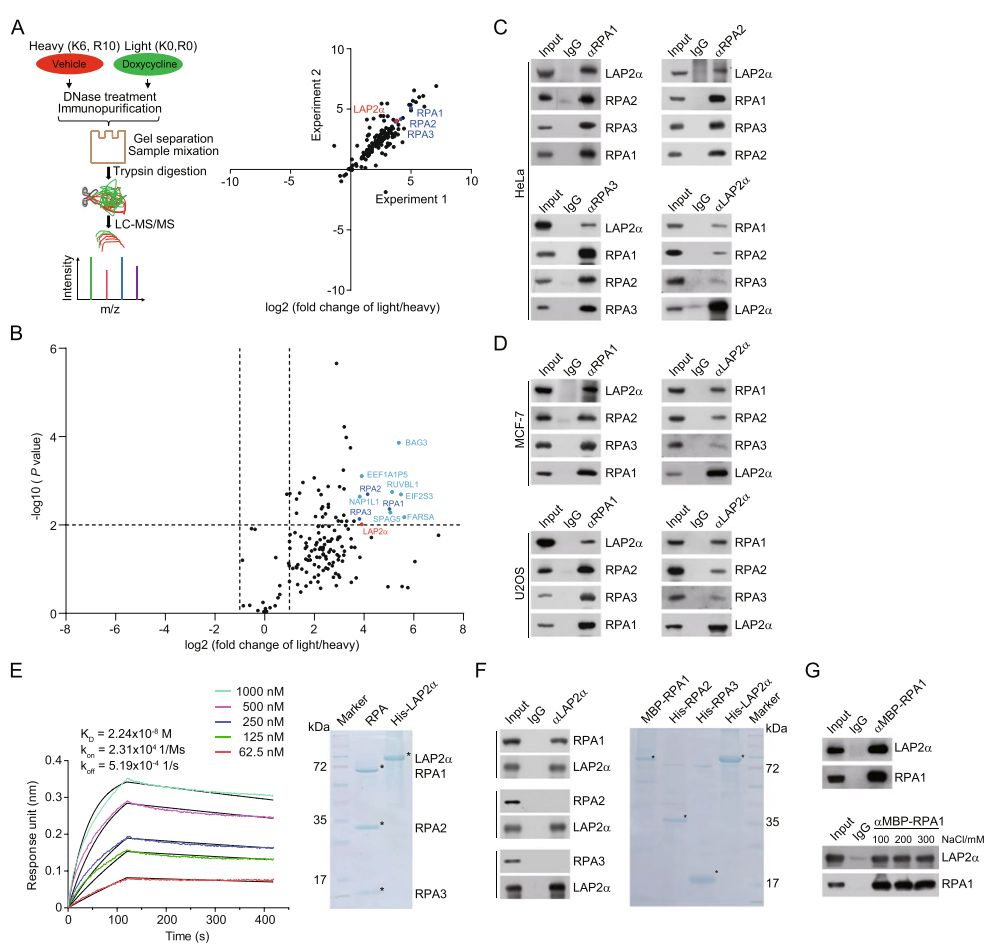

Fig. 1 RPA is physically associated with LAP2a. A SILAC-based quantitative mass spectrometry analysis of RPA1-containing protein complexes with HeLa cells that allow doxycycline (Dox)-inducible expression of stably integrated FLAG-RPA1. Control cells were labelled with "heavy isotopic lysine and arginine" (K6R10) and the cells under Dox (1 ng/ $\mu$ l) treatment were labelled with "light isotopic lysine and arginine" (KORO). DNase (300 U/ml)-treated cellular extracts were immunopurified with anti-FLAG affinity beads and eluted with FLAG peptide. The eluates were desalted by gel separation and mixed for digestion followed by mass spectrometry analysis. Biological duplicate experiments were performed. B Volcano plot showing the relative enrichment of RPA1-containing protein complex from SILAC-based quantitative mass spectrometry. Each point represents the potential interactor, and the 11 top candidates (fold change $>15$ and $P$ value $<0.01$ ) are indicated. $\mathbf{C}$ Co-immunoprecipitation analysis of the interaction between LAP2 $a$ and RPA. Whole-cell lysates from HeLa cells were pre-treated with DNase followed by immunoprecipitation and immunoblotting with antibodies against the indicated proteins. a, anti-. D Co-immunoprecipitation analysis of the interaction between LAP2 $\mathrm{a}$ and RPA. Whole-cell lysates from MCF-7 and U2OS cells were pre-treated with DNase followed by immunoprecipitation and immunoblotting with antibodies against the indicated proteins. $\mathbf{E}$ Analysis of the binding affinity of recombinant RPA purified from bacteria cells and His-tagged LAP2a purified from insect cells by biolayer interferometry (BLI) assay. The black line indicates fitted curves and the color traces represent raw data. Data are representative of two independent experiments. RPA1, RPA2, and RPA3 of the RPA complex were co-purified and examined by Coomassie brilliant blue staining. F Co-immunoprecipitation analysis of the association of individual recombinant RPA subunit with LAP2a. All proteins were individually purified from insect cells. G Co-immunoprecipitation analysis of the association of recombinant RPA1 with LAP2a in buffer with an increasing amount of ionic strength

To validate the interaction between LAP2 $\alpha$ and RPA and to gain a molecular insight into the assembly of the LAP2 $\alpha /$ RPA complex, real-time binding of LAP2 $\alpha$ to RPA was monitored by biolayer interferometry (BLI) with recombinant proteins. After immobilizing RPA on the surface of a sensor chip, various concentrations of recombinant LAP2 $\alpha$ were injected over the immobilized RPA to measure the binding affinities. This quantitative biophysical analysis shown as representative sensorgrams with global fitting curves revealed that LAP2 $\alpha$ strongly binds to RPA with an apparent dissociation constant $\left(K_{\mathrm{D}}\right)$ of $22.4 \mathrm{nM}$ (Fig. 1E). Next, we examined the binding of LAP2 $\alpha$ to each recombinant component of RPA with pull-down assays. The results indicated that only RPA1 could 
directly and strongly interact with LAP2 $\alpha$ (Fig. 1F, G). Collectively, these results suggest that LAP2 $\alpha$ is physically associated with RPA through directly binding to RPA1.

\section{Key determinants for LAP2a-RPA binding}

To understand the molecular details of the association of LAP2 $\alpha$ with RPA1, domain deletion mutants of RPA1 were generated. Co-immunoprecipitation experiments with RPA1 mutants followed by immunoblotting with RPA2 and LAP2 $\alpha$ revealed that the DBD-A domain of RPA1 was specifically required for the interaction of RPA1 with LAP2 $\alpha$, while the DBD-C domain deletion variant that could not bind RPA2 was still able to interact with LAP2 $\alpha$ (Fig. 2A). These results further confirmed that RPA1 functions to directly engage LAP $2 \alpha$ into the RPA complex. Reciprocally, co-immunoprecipitation assays indicated that the linker region, which is conserved across different species (Fig. 2B, C), was responsible for the interaction of LAP2 $\alpha$ with RPA1 (Fig. 2D, E), and the $\mathrm{C}$-terminal region of LAP2 $\alpha$ was responsible for its binding to Lamin A/C and $\mathrm{pRB}$ as previously reported [36] (Fig. 2D). Further chopping of the linker region of LAP2 $\alpha$ showed that the region spanning from amino acid 76 to 89 (76-89) was required for the interaction of LAP2 $\alpha$ with RPA1 (Fig. 2F), and co-immunoprecipitation assays with deletion mutants suggested that the fragment 90-109 was also essential in LAP2 $\alpha-R P A 1$ binding (Fig. 2G).

We then applied site-directed mutagenesis and measured the effect of these mutations on the interaction between LAP $2 \alpha$ and RPA1. The results indicated that Arg 86 (R86) and Arg 88 (R88) were critically required for LAP2 $\alpha-R P A 1$ binding, as alanine $(\mathrm{LAP} 2 \alpha / 2 \mathrm{RA})$, glutamate $(\mathrm{LAP} 2 \alpha / 2 \mathrm{RE})$, or glutamine (LAP2 $\alpha / 2 \mathrm{RQ})$ substitution dramatically compromised the interaction (Fig. $2 \mathrm{H}$ ). Meanwhile, we found these variants had minor effect on the association of LAP2 $\alpha$ with Lamin A/C or pRB (Fig. 2H). Since all of the mutations among 90-109 had little effect on LAP2 $\alpha$-RPA1 partnering (Fig. $2 \mathrm{H}$ ), we propose that 76-89 in the linker region of LAP2 $\alpha$ likely binds to RPA1 directly, while 90-109 may contribute to the interaction indirectly. Moreover, pull-down experiments with recombinant proteins demonstrated that wild type LAP2 $\alpha$ (LAP2 $\alpha / w \mathrm{t})$, but not LAP2 $\alpha / 2$ RE, could bind DBD-A or RPA (Fig. 2I, J). These results suggest R86/R88 act as key determinants for the interaction of LAP2 $\alpha$ with RPA.

\section{LAP2a promotes the loading of RPA onto damaged chromatin}

Since the ssDNA-RPA platform constitutes a key physiological signal in orchestrating DNA replication and repair, we wondered whether LAP2 $\alpha$ could facilitate RPA deposition onto ssDNA in vivo. First, isolation of proteins on nascent DNA (iPOND) assay was performed to analyze chromatin factors bound to nascent replicating forks in the absence or presence of hydroxyurea (HU), a reversible inhibitor of ribonucleotide reductase that stalls DNA replication forks by reducing cellular deoxynucleotide pools [38]. The results revealed that, in $\operatorname{Lap} 2 \alpha$ knockout $\left(\operatorname{Lap} 2 \alpha^{-/-}\right)$mouse embryonic fibroblast cells (MEFs), RPA loading at EdU-labelled nascent ssDNA of replication forks was dramatically disrupted under HU treatment (Fig. 3A, B). We showed that Lap $2 \alpha$ knockout or LAP $2 \alpha$ knockdown only had a moderate effect on RPA deposition in the absence of HU treatment (Fig. 3A, B and Additional file 2: Figure S1A), suggesting LAP $2 \alpha$ may play a limited role in RPA loading when DNA replicates. By 


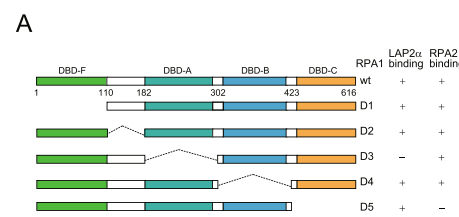

C

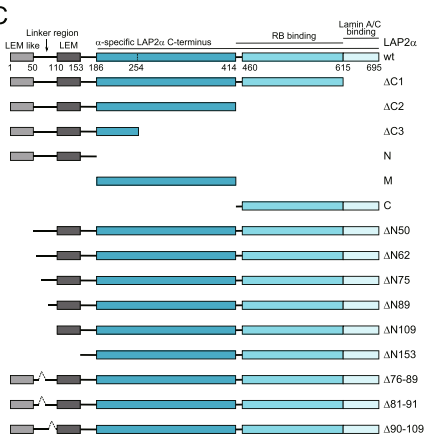

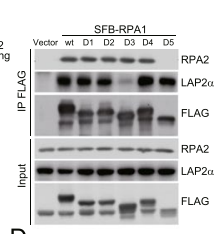

D
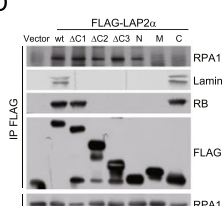

: $=$ =ニニニニニ ニニニニニニニ Lan

$\mathrm{F}$

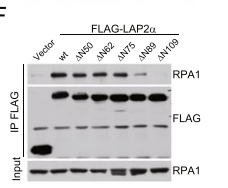

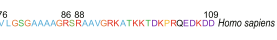

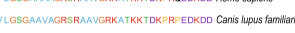

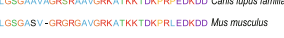

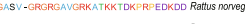

E FLAG-LAP2

FLAG-LAP2

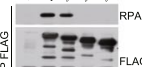

$\stackrel{a}{a}$
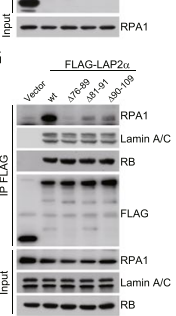

$\mathrm{H}$

I
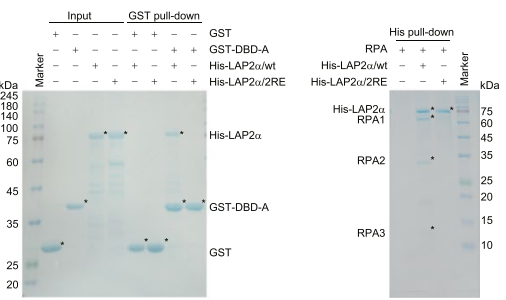

Fig. 2 Key determinants for LAP2a-RPA binding. A Whole-cell lysates from HeLa cells expressing $S$ protein-FLAG-Streptavidin binding peptide (SFB)-tagged RPA1 deletions were immunoprecipitated and immunoblotted with antibodies against the indicated proteins. The RPA1 domain and deletion mutants annotated with residue numbers are shown. DBD represents DNA-binding domain. B Schematic representation of the evolutionarily conserved residues in the linker region of LAP2a. C Schematic representation of the domain structure of LAP2 $a$ and its variants. The domains and truncated or deleted variants are marked with the indicated residue numbers. The LEM (LAP2, emerin, MAN1) domain, LEM-like domain, LAP2a-specific C-terminus, and RB or Lamin A/C binding regions are shown. N, N-terminus from amino acid 1 to 185; M, middle region from 186 to 414; C, C-terminus from 415-695. $\triangle N 50$, residues from $1-50$ are deleted; $\triangle 76-89$, residues from 76 to 89 are deleted; and so forth. D-F Whole-cell lysates from HeLa cells expressing FLAG-GFP-tagged LAP2a truncation mutants were immunoprecipitated and immunoblotted with antibodies against the indicated proteins. G Whole-cell lysates from HeLa cells expressing FLAG-GFP-tagged LAP2a deletion mutants were immunoprecipitated and immunoblotted with antibodies against the indicated proteins. H FLAG-tagged LAP2a variants were transfected into HeLa cells followed by co-immunoprecipitation and immunoblotting analysis. RK>AA (90-109) represents that all arginine and lysine residues from amino acid 90 to 109 of LAP2a are simultaneously replaced by alanine, and so forth. I GST pull-down assays with recombinant GST-DBD-A and His-tagged LAP2a variants. GST-DBD-A and His-tagged LAP2a were purified from bacteria cells and insect cells, respectively. $\mathbf{J}$ His pull-down assays with recombinant His-tagged LAP2a variants and RPA. RPA (including RPA1, RPA2, and RPA3) and His-tagged LAP2a variants were purified from bacteria cells and insect cells, respectively. The asterisks indicate the recombinant proteins stained by Coomassie brilliant blue

contrast, the iPOND signal of RPA was markedly reduced in cells expressing siRNA against CDC45 (Additional file 2: Figure S1A), which has been proposed to directly load RPA on the emerging nascent ssDNA at replication fork [32]. The binding of DNA sliding clamp proliferating nuclear antigen A (PCNA), a processivity factor for the elongation of nascent DNA strands at replication forks, was examined to monitor replication fork activity and it was largely unaffected in $\operatorname{Lap} 2 \alpha^{-} /^{-}$cells without HU treatment (Fig. 3A, B). Unlike CDC45, only a minor fraction of LAP2 $\alpha$ is associated 


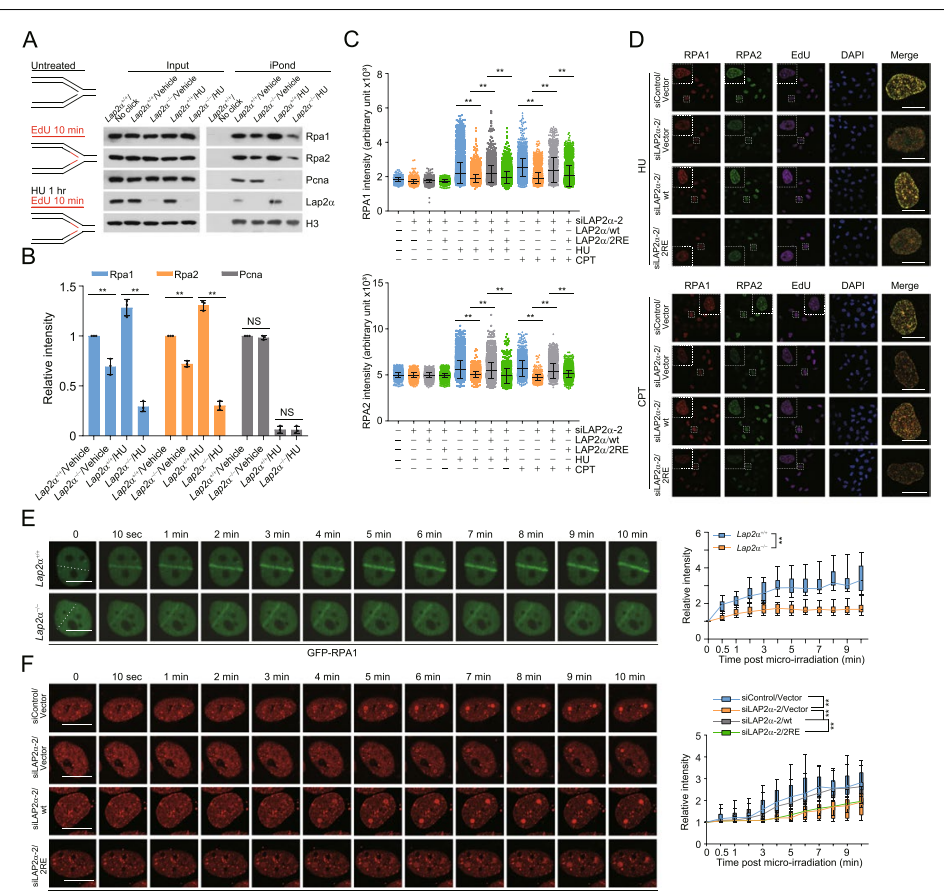

Fig. 3 LAP2a promotes the loading of RPA onto damaged chromatin. A Proteins associated with replication forks were isolated by iPOND as described in "Methods" and detected by immunoblotting. Lap $2 a^{+/+}$or

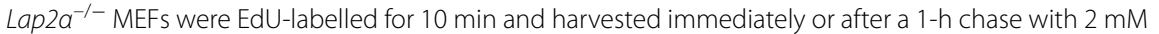
HU. B Quantitative analysis of Rpa1, Rpa2, and Pcna binding to EdU-labelled nascent replication forks with iPOND assays. The relative intensity of chromatin bound factors to histone $\mathrm{H} 3$ was normalized against that in vehicle-treated control cells. C High content imaging analysis of foci intensity of RPA1 and RPA2 in U2OS cells. pLenti-vector, LAP2a/wt or LAP2a/2RE stably integrated U2OS cells were transfected with control or LAP2a 3'UTR siRNA (siLAP2a-2) and treated with $2 \mathrm{mM} \mathrm{HU}$ for $4 \mathrm{~h}$ or $1 \mu \mathrm{M}$ CPT for $2 \mathrm{~h}$ followed by pre-extraction, fixation, immunostaining, and high content microscopy analysis. Before collection, cells were labelled with EdU for $1 \mathrm{~h}$. D Representative images from $\mathbf{C}$ collected by confocal microscopy. E Laser micro-irradiation (IR) (50\% laser energy) followed by live cell imaging analysis of GFP-RPA1 recruitment kinetics in GFP-RPA1 expressing Lap $2 \mathrm{a}^{+/+}$or Lap2a ${ }^{-/-}$MEFs. Fluorescence intensities in micro-irradiated areas relative to the nuclear background were quantified $(n>20)$. $\mathbf{F}$ pLenti-vector, LAP2a/wt or LAP2a/2RE stably integrated U2OS cells were co-transfected with DsRed-RPA1 and control siRNA or LAP2a 3'UTR siRNA followed by Hoechst sensitization, micro-IR $(405 \mathrm{~nm})$ and live cell imaging analysis. Fluorescence intensities in micro-irradiated areas relative to the nuclear background were quantified $(n>20)$. Data are mean \pm SDs for $\mathbf{B}, \mathbf{E}$, and $\mathbf{F}$ from biological triplicate experiments, and $\mathbf{C}$ from biological duplicate experiments. ${ }^{*} P<0.01$; NS, not significant; one-way ANOVA for B; Mann-Whitney test for $\mathbf{C}$; two-way ANOVA for $\mathbf{E}$ and $\mathbf{F}$. Scale bar, $10 \mu \mathrm{m}$

with unperturbed forks undergoing replication, but it becomes more abundant when forks stall (Fig. 3A and Additional file 2: Figure S1A). Possibly, there is a transient interaction between LAP $2 \alpha$ and the replicating chromatin, while LAP $2 \alpha$-directed RPA loading in HU-challenged cells is controlled by certain factor that functions to actively recruit the LAP $2 \alpha-\mathrm{RPA}$ complex or retain LAP $2 \alpha$ on damaged chromatin.

To further investigate the role of LAP $2 \alpha$ in promoting RPA deposition in stressed condition, ssDNA-pull-down assay was performed with nuclear extracts from wild type $\left(\operatorname{Lap} 2 \alpha^{+} /^{+}\right)$and $\operatorname{Lap} 2 \alpha^{-/-}$MEFs to assess ssDNA-RPA formation. In this assay, it has been proven that 70-nucleotide (nt) length of ssDNA may, to certain extent, mimic perturbed replication fork and will efficiently activate replication stress response $[39,40]$. The results indicated that Lap $2 \alpha$ deficiency dramatically impaired 
RPA loading (Additional file 2: Figure S1B). The same is true when ssDNA-pull-down assays were performed with LAP2 $\alpha$-knockdown cells (Additional file 2: Figure S1C). Furthermore, we demonstrated that LAP $2 \alpha / w t$, but not LAP $2 \alpha / 2$ RE, could efficiently restore RPA binding in LAP2 $\alpha$-depleted cells (Additional file 2: Figure S1D). At the time of analysis, Lap $2 \alpha$ knockout or LAP2 $\alpha$ knockdown did not significantly alter the cell-cycle profile (Additional file 2: Figure S1E and S1F). In agreement with the observations from iPOND assays under HU-challenged condition, we found LAP2 $\alpha$ as well as its variant LAP $2 \alpha / 2$ RE could be also pulled down with RPA by biotinylated-ssDNA (Additional file 2: Figure S1D).

Then, we examined whether LAP2 $\alpha$ affects RPA foci formation on stalled or collapsed replication forks. As shown in Fig. 3C and D, discrete foci of RPA1 and RPA2 are observed upon HU treatment in the nuclei of EdU-positive cells. Remarkably, LAP2 $\alpha$ depletion impaired the recruitment of RPA1 and RPA2 to the blocked forks, and the defective RPA foci formation could be significantly overcome by overexpression of LAP $2 \alpha / w t$, but not LAP $2 \alpha / 2$ RE (Fig. 3C, D). Similar results were obtained when these cells were challenged with camptothecin (CPT) (Fig. 3C, D), which poisons Topoisomerase I and leads to stalling or collapse of replication forks [41, 42]. Next, we tested whether LAP2 $\alpha$ is required for RPA loading onto blocked replication fork using the Lac operon-Lac repressor (LacO-LacI) tethering system [43]. In this approach, the stably integrated $\mathrm{LaCO}$ operator is inherently and tightly bound by LacI, and the LacO-LacI nucleating complex poses an obstacle to DNA polymerase progression, leading to site-specific replication fork blockage [43, 44]. A robust accumulation of RPA around the LacO arrays was observed in cells expressing an mCherry-LacI fusion protein, manifested by the co-localization of RPA1 with mCherry-LacI (Additional file 2: Figure S1G). LAP2 $\alpha$ knockdown prominently compromised RPA accumulation on mCherry-LacI marked blocked replication site, and this effect could be reverted by forced expression of LAP $2 \alpha / w t$, but not LAP2 $\alpha / 2$ RE (Additional file 2: Figure S1G). These results further support the argument that LAP2 $\alpha$ is essentially required for RPA deposition in responding to replication stress.

Next, we wondered whether LAP2 $\alpha$ could control RPA loading to DNA lesions. To this end, breaks were induced by laser micro-point equipped with 365-nm UV laser beam that is generally used to create single-strand breaks (SSBs) and DSBs, and the kinetics of RPA deposition was first evaluated in $\operatorname{Lap} 2 \alpha^{+} /^{+}$and $\operatorname{Lap} 2 \alpha^{-} /^{-}$MEFs. Live cell imaging followed by confocal microscopy analysis indicated that Lap $2 \alpha$ deficiency resulted in a low efficiency of RPA loading, evidenced by a mild reduction of laser stripe formation of GFP-tagged RPA1 at early response phase and a remarkably weakened pattern at late time points (Fig. 3E and Additional file 2: Figure $\mathrm{S} 1 \mathrm{H}$ ). The increasing difference of RPA intensity at laser stripes from early to late time points in wild type versus knockout cells (Additional file 2: Figure $\mathrm{S} 1 \mathrm{H}$ ), suggests that there could be a differential extent of requirement of LAP2 $\alpha$ for RPA loading during a progressive DNA end resection-coupled RPA deposition process. Furthermore, we showed that LAP $2 \alpha /$ wt could significantly restore LAP $2 \alpha$ depletion-associated defects of RPA1 recruitment, whereas LAP2 $\alpha / 2$ RE failed to do so (Fig. 3F). Taken together, our results support the notion that LAP2 $\alpha$ acts as an essential regulator in 
promoting RPA association with damaged chromatin in the presence of replication stress or DNA breaks, indicating that LAP2 $\alpha$ may chaperone RPA in vivo.

Since all splicing variants of LAP2 share the same N-terminal region, we wondered whether other isoforms of LAP2 anchored at nuclear envelope act similarly as LAP2 $\alpha$. We first found LAP2 $\beta$ knockdown had marginal effect on site-specific RPA recruitment upon replication fork stalling (Additional file 2: Figure S1I). Moreover, rescue experiments indicated that the defects of RPA accumulation induced by LAP2 depletion could be efficiently restored by overexpression of siRNA-resistant LAP2 $\alpha$, but not that of LAP2 $\beta$ (Additional file 2: Figure S1J). Although we could not exclude the possibility that the inner membrane-associated LAP2s may function to facilitate RPA binding to ssDNA that is adjacent to nuclear envelope, these results suggest that LAP2 $\alpha$ is the major splicing variant of LAP2 that controls RPA loading in the nucleoplasm territories. Meanwhile, we examined whether Lamin A/C, which has been involved in DNA damage repair [45-47], plays a role in RPA loading. The results indicated that the recruitment of GFP-RPA1 was largely unaffected in $L m n a^{-1-}$ MEFs (Additional file 2: Figure S1K). Similar results were obtained when the foci formation of RPA1 and RPA2 were examined in Lmna ${ }^{-1-}$ MEFs and Lamin A/C knockout U2OS cells (Additional file 2: Figure S1L and S1M). Consistently, co-immunoprecipitation experiments showed that Lamin A/C could not be precipitated by RPA1 (Additional file 2: Figure S1N). Thus, we propose that Lamin A/C is not involved in RPA loading and LAP2 $\alpha$ functions independently of Lamin $\mathrm{A} / \mathrm{C}$ in this process.

\section{LAP2a maintains DNA replication integrity through loading RPA onto ssDNA}

Because RPA functions as an essential module in binding ssDNA and protecting stalled forks from nucleolytic degradation, we wondered whether LAP2 $\alpha$ plays a role in DNA replication integrity. To this end, DNA fiber analysis was carried out to assess replication fidelity at single-molecule resolution. First, $\operatorname{Lap} 2 \alpha^{+/+}$or $\operatorname{Lap} 2 \alpha^{-/-}$MEFs were labelled sequentially with nucleoside analogs 5'-iodo-2-deoxyuridine (IdU) and 5'-chloro-2-deoxyuridine (CldU), and the track lengths of single DNA fibers were measured following HU treatment. The ratios of the lengths of adjacent CldU and IdU approximated unity under HU treatment in Lap $2 \alpha^{+/+}$MEFs (Fig. 4A), indicating that the integrity of stalled forks is largely unaffected during prolonged replication stress. In contrast, the CldU/IdU ratios were significantly reduced in HU-treated Lap $2 \alpha^{-1-}$ MEFs (Fig. 4A), implying a defect in stalled fork protection. This was prevented by addition of the nuclease inhibitor mirin (Fig. 4A). Identical results were obtained upon analysis of fibers from LAP2 $\alpha$ knockdown human cells (Fig. 4B). Importantly, we showed LAP2 $\alpha$ depletion-associated fork degradation could be successfully compensated by forced expression of LAP $2 \alpha$ / wt, but not by LAP2 $\alpha / 2$ RE that is defective in binding and loading RPA (Fig. 4B). These results are consistent with the role of RPA in fork protection $[17,48]$ and raised the possibility that LAP $2 \alpha$ functions to protect nascent strands on stalled replication forks via facilitating RPA deposition.

To further examine whether LAP $2 \alpha$ is needed to maintain replication integrity, we analyzed the ability of LAP2 $\alpha$-deficient cells to recover from HU-induced replication arrest. After the release from $\mathrm{HU}$ treatment, $\gamma \mathrm{H} 2 \mathrm{AX}$ foci largely disappeared from control cells but persisted in Lap $2 \alpha^{-1-}$ MEFs (Fig. 4C). Next, comet assays demonstrated 
A

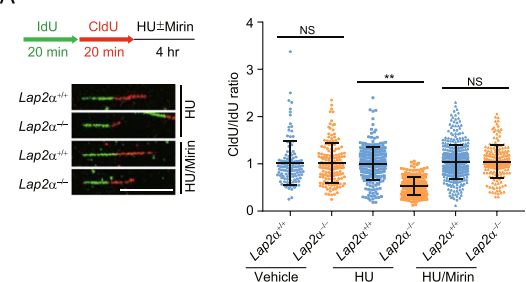

C

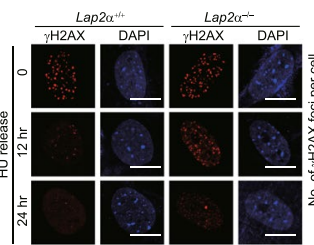

Lap $2 \alpha^{-\alpha^{-}}$

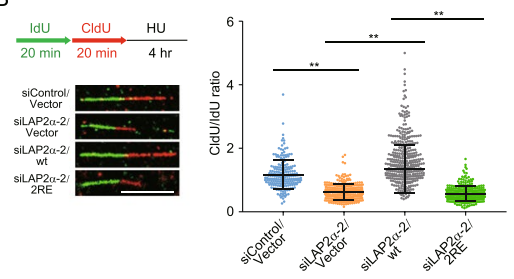

D
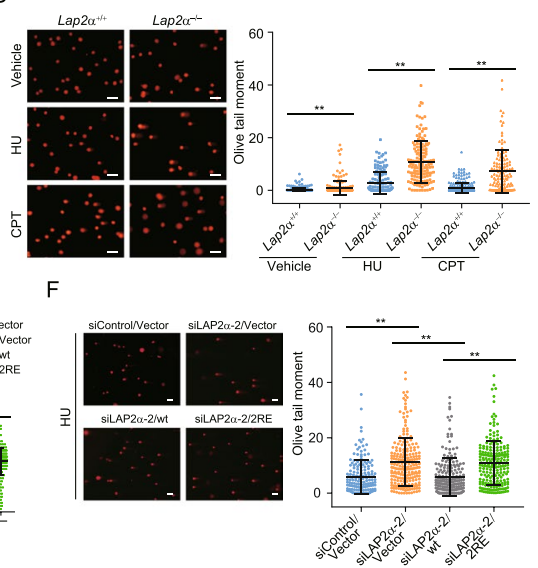

Fig. 4 LAP2a maintains DNA replication integrity through loading RPA onto ssDNA. A DNA fiber assay with

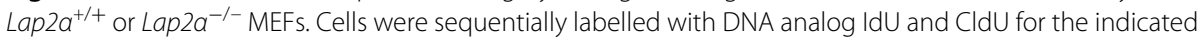
time followed by $\mathrm{HU}(4 \mathrm{mM}, 4 \mathrm{~h})$ treatment in the absence or presence of mirin $(100 \mu \mathrm{M}, 4 \mathrm{~h})$. The ratios of $\mathrm{CldU}$ and IdU length were calculated in each treatment $(n>150)$. Scale bar, $10 \mu \mathrm{m}$. B pLenti-vector, LAP2a/ wt or LAP2a/2RE stably integrated U2OS cells were transfected with control siRNA or LAP2a 3'UTR siRNA and experiments analogous to $\mathbf{A}$ were performed $(n>150)$. Scale bar, $10 \mu \mathrm{m}$. C Lap $2 a^{+/+}$or Lap2a ${ }^{-/-}$MEFs were treated with $2 \mathrm{mM} \mathrm{HU}$ for $16 \mathrm{~h}$ and released followed by $\mathrm{\gamma H} 2 \mathrm{AX}$ staining and confocal microscopy inspection. The foci number of $\mathrm{YH} 2 \mathrm{AX}$ per cell in each treatment was quantified $(n>100)$. Scale bar, $10 \mu \mathrm{m}$. D Accumulation of damaged DNA was examined and quantified with alkaline comet assay in $\mathrm{HU}(2 \mathrm{mM}$,

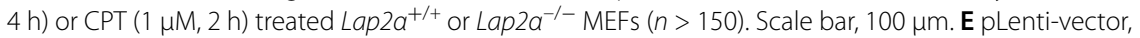
LAP2a/wt or LAP2a/2RE stably integrated U2OS cells were transfected with control siRNA or LAP2a 3'UTR siRNA and experiments analogous to $\mathbf{C}$ were performed $(n>150)$. Scale bar, $10 \mu \mathrm{m}$. $\mathbf{F}$ pLenti-vector, LAP2a/ wt or LAP2a/2RE stably integrated U2OS cells were transfected with control siRNA or LAP2a 3'UTR siRNA followed by HU treatment and experiments analogous to $\mathbf{D}$ were performed $(n>300)$. Scale bar, $100 \mu \mathrm{m}$. Data are mean \pm SDs for $\mathbf{A}$ and $\mathbf{B}$ from biological duplicate experiments, and $\mathbf{C}-\mathbf{F}$ from biological triplicate experiments. ${ }^{*} P<0.05$; ${ }^{* *} P<0.01$; NS, not significant; Mann-Whitney test

that Lap $2 \alpha$-knockout cells contained markedly elevated levels of DNA breaks under either HU or CPT treatment (Fig. 4D). A moderate upregulation of damaged DNA was also observed in Lap $2 \alpha$-knockout cells without exogenous stress (Fig. 4C, D). Furthermore, we showed that LAP2 $\alpha$-knockdown associated accumulation of DNA damage in stressed condition could be efficiently reduced by forced expression of LAP2 $\alpha / w t$, but not that of LAP $2 \alpha / 2 R E$ (Fig. 4E, F). Thus, we propose that LAP2 $\alpha$-promoted RPA deposition is critically required for cells to cope with replication stress thus maintaining replication fidelity.

\section{LAP2a-promoted RPA loading is required for ATR activation and homologous recombination}

Next, we tested whether LAP2 $\alpha$ plays a role in RPA-directed ATR activation upon replication stress. Immunoblotting analysis indicated that LAP2 $\alpha$ depletion markedly 
reduced the phosphorylation level of RPA2 S33 and CHK1 S345, which are substrates of ATR and generally used to monitor ATR activity (Fig. 5A). Unlike LAP2 $\alpha / w t$, LAP $2 \alpha / 2 R E$ overexpression was incapable of reverting LAP2 $\alpha$-depletion-associated defects of ATR activation in LAP2 $\alpha$-knockdown cells (Fig. 5A). Similar results were obtained when the intensity of RPA2 S33 phosphorylation was examined with fluorescent immunostainings in HU-challenged EdU-positive cells (Additional file 2: Figure S2A). Meanwhile, we found Lap $2 \alpha^{-1-}$ MEFs in normal conditions showed mildly reduced fork elongation rates, accompanied by a decrease in the symmetry of bidirectional replication forks and an increase in new origin firing (Fig. 5B). This is consistent with the understanding that ATR inhibition leads to compromised replication fork elongation and unscheduled replication timing [49].

To assess the role of LAP2 $\alpha$-promoted RPA loading in DSB repair, we then examined the foci formation of RAD51 that functions downstream of RPA [22]. As shown

A

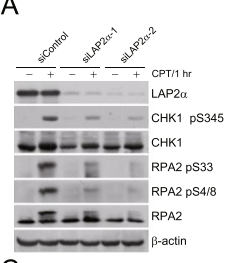

C

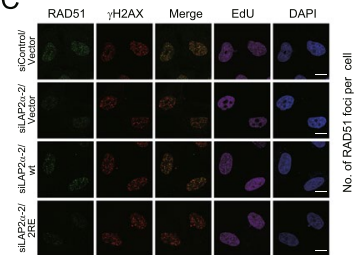

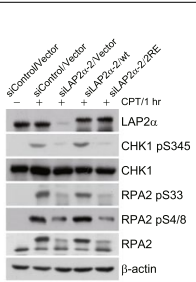

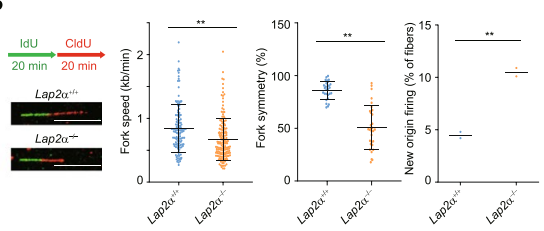

D

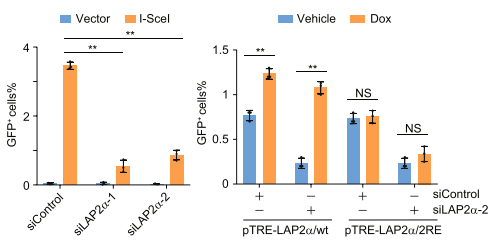

E

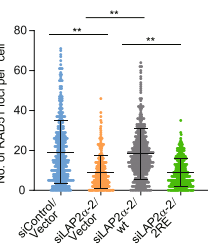

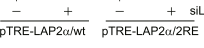

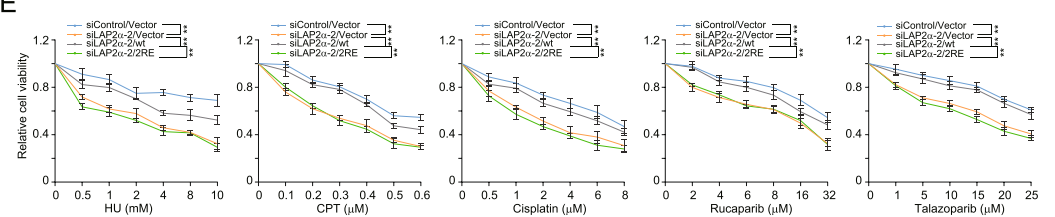

Fig. 5 LAP2a-promoted RPA loading is required for ATR activation and homologous recombination. A Immunoblotting analysis of ATR kinase activity. Control U2OS cells and LAP2a/wt or LAP2a/2RE stably integrated U2OS cells were transfected with indicated siRNAs and in the absence or presence of CPT (1 $\mu \mathrm{M}, 1 \mathrm{~h})$. The cellular extracts were collected to examine CPT-induced phosphorylation events. B DNA fiber assay with Lap $2 a^{+/+}$or Lap $2 a^{-/-}$MEFs. Cells were sequentially labelled with DNA analog IdU and CldU for the indicated time. Fork speed $(n>120)$ and fork symmetry $(n>25)$ were determined by measuring the length of $\mathrm{CldU}$ track, and the percentage of new origins was quantified with only CldU staining fibers. $\mathbf{C}$ Immunostaining and confocal microscopy analysis of RAD51 foci formation. LAP2a/wt or LAP2a/2RE stably integrated U2OS cells were transfected with LAP2a 3'UTR siRNA, exposed to IR (4 Gy) and cultured for $3 \mathrm{~h}$ followed by $1 \mathrm{~h}$ EdU labelling before collection. The foci number of RAD51 per EdU-positive cell in each group was quantified ( $n>150$ ). D Homologous recombination efficiencies monitored by DR-GFP reporter assays. Control DR-GFP U2OS cells and DR-GFP U2OS cells that allow for Dox-inducible expression of stably integrated pTRE-LAP2a/wt or PTRE-LAP2a/2RE were co-transfected with HA-I-Scel and the indicated siRNAs. Cells were treated with vehicle or Dox $(1 \mathrm{ng} / \mu \mathrm{l})$ for $48 \mathrm{~h}$ to induce the expression of LAP2a/wt or LAP2a/2RE. The proportions of GFP-positive cells were determined by flow cytometry. $\mathbf{E}$ Survival analysis of U2OS cells expressing LAP2a 3'UTR siRNA and LAP2a/wt or LAP2a/2RE under different drug treatment. Data are mean \pm SDs for $\mathbf{B}$ from biological duplicate experiments, and $\mathbf{C}$-E from biological triplicate experiments. ${ }^{*} P<0.01$; NS, not significant; Mann-Whitney test for $\mathbf{B}$ and $\mathbf{C}$; one-way ANOVA for $\mathbf{D}$; two-way ANOVA for $\mathbf{E}$. Scale bar, 10 $\mu \mathrm{m}$ 
in Fig. 5C, LAP2 $\alpha$ depletion resulted in a significant impairment of RAD51 foci formation upon irradiation, and this effect could be faithfully reverted by LAP2 $\alpha / w t$, but not LAP2 $\alpha / 2$ RE. Meanwhile, we found that, in LAP2 $\alpha$-deficient cells, the expression of RAD51 and RPA was not affected (Additional file 2: Figure S2B). Importantly, we showed LAP2 $\alpha$ depletion had minor effect on BrdU foci or laser stripe formation under non-denaturing conditions (Additional file 2: Figure S2C and Figure S2D), excluding the possibility that LAP2 $\alpha$-promoted RPA deposition on DNA damage sites is a secondary effect of enhanced DNA end resection. Next, the DNA repair efficiency was monitored in DR-GFP U2OS cells, a well-known tool for detecting HR activity manifested by alterations of percentage of GFP-positive cells $[50,51]$. The results showed LAP2 $\alpha$ knockdown significantly compromised the efficiency of HR (Fig. 5D). In support of the importance of the functional link between LAP2 $\alpha$ and RPA, we found that overexpression of LAP $2 \alpha / w t$, but not LAP $2 \alpha / 2 R E$, could evidently compensate the HR defects in LAP2 $\alpha$-knockdown cells (Fig. 5D and Additional file 2: Figure S2E). Collectively, these data indicate that LAP2 $\alpha$-promoted RPA loading plays an important role in HR repair of DSBs.

We next examined the effect of LAP $2 \alpha$ depletion on cell survival upon replication stress induced DNA damage. The results indicated that LAP2 $\alpha$-depleted cells were more vulnerable to $\mathrm{HU}, \mathrm{CPT}$, or cisplatin (a DNA crosslinker that will generate replication stress) (Fig. 5E). Since long-term treatment with these agents will lead to fork collapse and the repair of resultant DSBs relies on HR, we envisioned that the hypersensitivity of LAP2 $\alpha$-deficient cells reflects an additive effect of fork instability, ATR inactivation, and HR deficiency. As cells with defects in HR or ATR signalling are synthetically lethal with poly(ADP-ribose)-polymerase (PARP) inhibition [52-54], we wondered whether cells lacking LAP $2 \alpha$ are hypersensitive to PARP inhibitors (PARPis). Indeed, LAP2 $\alpha$-depleted cells exhibited a much lower survival rate following rucaparib or talazoparib treatment (Fig. 5E). Furthermore, cell survival analysis confirmed that LAP2 $\alpha$-protecting cells from replication stress-associated DNA damage depends on its association with and loading of RPA, as LAP $2 \alpha / 2 \mathrm{RE}$ failed to compensate LAP2 $\alpha$ depletion-induced effects (Fig. $5 \mathrm{E}$ ). Collectively, these data suggest that LAP2 $\alpha$ is critically involved in responding to replication stress and repair of DNA lesions via actively promoting RPA deposition.

\section{LAP2 $\alpha$ is engaged into damaged chromatin in a PARP1-dependent manner}

As LAP $2 \alpha$ could be pulled down by ssDNA-nucleated protein complexes, and it is present on nascent ssDNA upon HU challenging, we suspected that LAP2 $\alpha$ is able to localize to damaged chromatin. Indeed, confocal microscopy analysis confirmed that LAP $2 \alpha$ was recruited to mCherry-LacI and RPA marked blocked forks (Additional file 2: Figure S3A). Meanwhile, we showed that GFP-tagged LAP2 $\alpha$ rather than LAP2 $\beta$ could form evident micro-IR path (Additional file 2: Figure S3B). In agreement with the observations from ssDNA-pull-down assays (Additional file 2: Figure S3C), endogenous LAP2 $\alpha$ was co-localized with $\gamma \mathrm{H} 2 \mathrm{AX}$ and RPA1 and it departed from DNA lesions progressively (Additional file 2: Figure S3D and S3E). Then, we sought to explore whether RPA recruits LAP2 $\alpha$ to damaged chromatin. Interestingly, RPA1 depletion has minor effect on the engagement of LAP2 $\alpha$ around damaged DNA sites (Additional file 2: Figure S3F), and LAP $2 \alpha / 2 R E$ behaved similarly as LAP $2 \alpha / w t$ when its recruitment kinetics was examined 
by micro-IR (Additional file 2: Figure S3G). This is consistent with the observations from ssDNA-pull-down assays, from which we can see LAP $2 \alpha / 2 R E$ could be also precipitated with ssDNA-RPA complex (Additional file 2: Figure S1D). These results imply that the physical contact between RPA and LAP2 $\alpha$ is likely lost once RPA has been deposited, and LAP $2 \alpha$ is finally removed from damaged chromatin after loading RPA.

To understand the underlying mechanisms that determine LAP2 $\alpha$ engagement, we generated HeLa cells stably expressing FLAG-LAP2 $\alpha$. Affinity purification and mass spectrometry analysis identified poly(ADP-ribose) polymerase 1 (PARP1) as a potential LAP2 $\alpha$-interacting protein, and the interaction was further confirmed by co-immunoprecipitation assays (Additional file 2: Figure S3H, S3I, and S3J; and Additional file 3: Table S2). Because one of the earliest events in DDR is the recruitment of PARP1 to diverse types of DNA damage, we hypothesized that PARP1 may act to localize LAP2 $\alpha$. Indeed, knockdown of PARP1 almost completely impaired laser stripe formation of LAP2 $\alpha$ (Additional file 2: Figure S3K). Cells were then treated with different PARPis including veliparib, rucaparib, and talazoparib to examine the contribution of the polymerase activity of PARP1. Surprisingly, the results indicated that, although the recruitment of CHD1L, a PAR binding protein [55], was severely abolished (Additional file 2: Figure S3L), the accrual of LAP2 $\alpha$ on damaged chromatin was not weakened, but strengthened, and the alterations were proportional to the chromatin trapping efficiency of these drugs [54], among which talazoparib had the strongest effect (Fig. 6A). These results indicate that LAP2 $\alpha$ is co-trapped with PARP1 at DNA lesions upon PARP inhibition and PARP1-promoted LAP2 $\alpha$ recruitment does not rely on the catalytic activity of PARP1.

Next, we showed that, in PARP1-depleted cells, the defective recruitment of LAP2 $\alpha$ could be significantly overcome by either wild type PARP1 (PARP1/wt) or PARylation activity-deficient PARP1 (PARP1/E988K) [56], in which glutamate 988 is replaced by lysine, further indicating that the enzymatic activity of PARP1 is dispensable for LAP2 $\alpha$ localization to damaged chromatin (Fig. 6B, C, and Additional file 2: Figure S3M). Meanwhile, ssDNA-pull-down experiments revealed that rucaparib treatment enhanced LAP $2 \alpha$ accumulation, PARP1 binding, and RPA loading, while LAP $2 \alpha$ depletion markedly compromised PARPi-induced RPA deposition (Fig. 6D). Furthermore, coimmunoprecipitation analysis demonstrated that PARPi treatment strengthened but not weakened the association of LAP2 $\alpha$ with PARP1 (Fig. 6E), and LAP2 $\alpha$ could equally bind to PARP1/wt and PARP1/E988K (Additional file 2: Figure S3N). Consistently, PARP1 knockdown weakened the loading of RPA on laser-generated DNA lesions or perturbed replication fork (Additional file 2: Figure S3O and S3P). Taken together, these results indicate that PARP1 promotes LAP2 $\alpha$ recruitment at damaged chromatin in a PARylation activity-independent manner and provides mechanistic insight of why LAP $2 \alpha$ is preferentially and selectively involved in damaged chromatin-associated RPA loading (Fig. 6F).

\section{Discussion}

A central regulator of eukaryotic DNA metabolism is ssDNA-RPA intermediate. However, it remains to be investigated that how the formation of ssDNA-RPA platform is controlled during replication stress or at DNA lesions. In this study, we characterized 


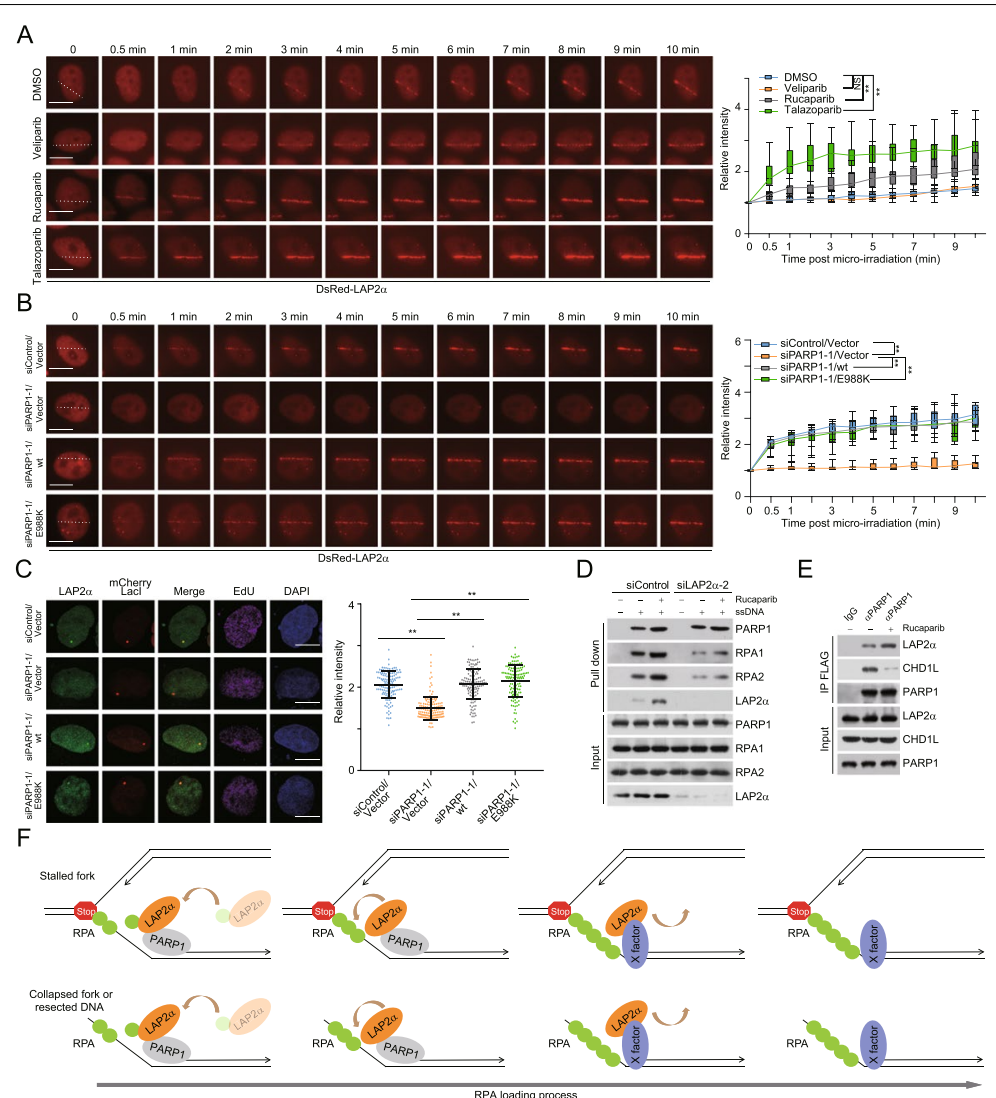

Fig. 6 LAP2a is engaged into damaged chromatin in a PARP1-dependent manner. A Laser micro-IR (35\% laser energy) followed by live cell imaging analysis of DsRed-LAP2a recruitment kinetics in PARP1 inhibitor (PARPi)-treated U2OS cells. DsRed-LAP2a expressing cells were pre-treated with different PARPis $(10 \mu \mathrm{M})$ for 4 $\mathrm{h}$ before laser micro-IR. Fluorescence intensities in micro-irradiated areas relative to the nuclear background were quantified $(n>20)$. B Laser micro-IR ( $50 \%$ laser energy) followed by live cell imaging analysis of DsRed-LAP2a recruitment kinetics in PARP1-knockdwon or -overexpression U2OS cells. pLenti-vector, PARP1/wt, or PARP1/E988K stably integrated U2OS cells were co-transfected with control siRNA or PARP1 3'UTR siRNA (siPARP1-1) and DsRed-LAP2a. Fluorescence intensities in micro-irradiated areas relative to the nuclear background were quantified $(n>20)$. C U2OS-LacO cells expressing mCherry-Lacl, PARP1 variants, and PARP1 3'UTR siRNA were labelled with EdU for $1 \mathrm{~h}$ before immunostaining and confocal microscopy analysis. The intensity of LAP2a foci in mCherry-Lacl and EdU-positive cells was quantified and normalized to the nuclear background ( $n>100$ ). D ssDNA-pull-down analysis of LAP2a accumulation, PARP1 binding, and RPA loading with nuclear extracts from LAP2a-knockdown U2OS cells under rucaparib (10 $\mu \mathrm{M}, 4 \mathrm{~h}$ ) treatment. $5^{\prime}$ biotin-labelled 70-nt ssDNA was used in pull-down assays. E Co-immunoprecipitation analysis of the interaction between LAP2a and PARP1 with cellular extracts from U2OS cells pre-treated with vehicle or rucaparib $(10 \mu \mathrm{M}, 4 \mathrm{~h})$. F A proposed regulatory model for PARP1 in directing LAP2a-promoted RPA loading at damaged chromatin. X-factor represents the molecular machinery that may function to retain the longer occupancy of LAP2a on damaged chromatin. Data are mean \pm SDs for $\mathbf{A}-\mathbf{C}$ from biological triplicate experiments. ${ }^{*} P<0.01$; NS, not significant; two-way ANOVA for $\mathbf{A}$ and $\mathbf{B}$; Mann-Whitney test for $\mathbf{C}$. Scale bar, $10 \mu \mathrm{m}$

LAP2 $\alpha$ as an essential regulator for RPA loading to ssDNA and this process requires physical contacts between LAP $2 \alpha$ and RPA1. Importantly, we characterized a separation-of-function mutant of LAP2 $\alpha$ and revealed that LAP2 $\alpha$-promoted RPA loading onto ssDNA and its role in genome stability are largely uncoupled from its pRB- or/ and Lamin $\mathrm{A} / \mathrm{C}$-associated functions, but specifically attributed to its physical and 
functional association with RPA. Interestingly, the selectivity of LAP2 $\alpha$-promoted RPA deposition on damaged chromatin is controlled by PARP1.

Since RPA is a critical regulator of DNA replication, it is important to understand whether LAP $2 \alpha$ could favor RPA binding to ssDNA in this process. Indeed, when LAP2 $\alpha$ was knocked out, a mild loss of RPA at active forks accompanying with a detectable accumulation of DNA breaks and a global reduction in DNA synthesis, albeit not dramatically, was observed under unstressed conditions in MEF cells. These results suggest that in normal cells, LAP2 $\alpha$ may, to a certain extent, contribute to the delivery of RPA and maintenance of genome integrity. This argument is consistent with LAP $2 \alpha$ expression feature: it is upregulated in proliferating cells and is downregulated upon cell-cycle exit or differentiation [34]. The moderate effect could be due to a weak activity of LAP2 $\alpha$ itself in replication-coupled RPA deposition, or due to compensation from other factors that play a role of importance in assistance of RPA loading. For example, CDC45, an essential factor for the initiation and elongation process of replication, is proposed to guarantee a seamless deposition of RPA on newly emerging ssDNA at the nascent replication forks [32]. Indeed, comparing to LAP2 $\alpha$ loss, CDC45 depletion exhibited more severe effects on RPA loading in replicating cells.

On the contrary, replication stress may potentially result in an accrual of ssDNA formation with larger stretches, where an instant requirement for more RPA binding must be fulfilled to maintain fork integrity [12]. Here, we revealed that LAP2 $\alpha$ is particularly important for RPA deposition in this circumstance, as the association of RPA with chromatin was significantly reduced at stalled or collapsed forks globally or at site-specific replication blockage upon LAP2 $\alpha$ loss. In agreement with these findings, we confirmed that LAP2 $\alpha$-promoted RPA loading is particularly important to uphold DNA replication integrity and genome stability. Considering that CDC45 is likely the major factor for RPA deposition on active replication forks [32] and LAP2 $\alpha$-promoted RPA loading is particularly important under replication stress, we propose that distinct molecular machineries could be utilized to control RPA binding to ssDNA under different DNA metabolic processes. Since the DBD-A domain of RPA interacts with both CDC45 and LAP2 $\alpha$, it is possible that CDC45-RPA and LAP2 $\alpha-$ RPA complexes directed by DBD-A are assembled in a context-dependent manner. As cellular LAP $2 \alpha$ is a multimeric protein channelled by its C-terminal region [36], it is worth characterizing whether this could affect its RPA loading activity in the future.

Excessive ssDNA, in the form of ssDNA gaps or resected ends of DSBs, can be generated by appropriate DNA nucleases at broken sites $[57,58]$. Consistently, we found that LAP2 $\alpha$ is also critically required for RPA loading to DNA lesions upon irradiation. In support of this, we showed that the activity of LAP2 $\alpha$ in loading RPA to ssDNA is critically essential for cells to repair damaged DNA via HR, implying that LAP2 $\alpha$ is generally involved in multiple aspects of RPA-dictated DNA metabolic signalling pathways. Interestingly, we showed LAP2 $\alpha$ is prominently localized to damaged chromatin in the occurrence of replication stress or DNA breaks, and the recruitment of LAP2 $\alpha$ is dependent on its physical association with PARP1. This, to certain extent, explains that why LAP $2 \alpha$ is preferentially responsible for RPA loading onto damaged chromatin. We noticed that LAP2 $\alpha$ is engaged into damaged chromatin in a dynamic manner, but what determines its clearance remains to be investigated. Considering the rapid turnover of PARP1 and PAR modification on damaged 
chromatin, we speculated that the relatively longer retention of LAP $2 \alpha$ must be controlled by other undefined factors. LAP $2 \alpha$ has been reported to be PARylated upon DNA damage $[59,60]$, while we demonstrated the enzymatic activity of PARP1 is largely irrelevant to LAP2 $\alpha$ engagement, implying that LAP2 $\alpha$, in particular of its PARylated form, may play additional roles in DDR. In contrast to Bártovás study [61], we showed both tagged and endogenous LAP2 $\alpha$ could be efficiently recruited to local DNA lesions, where it co-localized with $\gamma \mathrm{H} 2 \mathrm{AX}$ and RPA1. The discrepancy may arise either from different UV generator systems we took or from distinct cell types we utilized.

In view of the recently reported role of LAP2 $\alpha$ in transcriptional regulation and chromatin conformation alterations $[62,63]$, it is important to decipher whether LAP2 $\alpha$ 's activity in genome surveillance could be specifically attributed to RPA loading. Many of the established functions of LAP2 $\alpha$ map to its unique C-terminal region. For example, residues 415-615 of LAP2 $\alpha$ interact with pRB [64], and the extreme C-terminus from residue 616 to 693 binds to Lamin A/C [65]. LAP2 $\alpha$ determines the distribution of Lamin A/C in the nucleoplasm in general [37] and on distinct chromatin regions in specific [62], and the LAP2 $\alpha$-Lamin A/C-pRB complex has been shown to influence cell-cycle progression and to control the balance between cell proliferation and differentiation [37, 64]. Meanwhile, LEM-like domain of LAP2 $\alpha$ confers its interaction with transcription factor GLI1 [35], and LEM domain confers BANF1- or BAF-binding activity [36, 66]. In contrast to these binding themes, we revealed that the flexible liker between LEM-like domain and the LEM domain is responsible for RPA binding. Importantly, deletion of this linker region or mutation of the key residues for RPA association had marginal effect on the interaction of pRB or Lamin A/C with LAP2 $\alpha$, and LAP2 $\alpha / 2$ RE failed to efficiently bind and load RPA and could not compensate LAP2 $\alpha$ depletion-induced RPA loading defects and genome instability. Meanwhile, we found Lamin A/C could not be precipitated by RPA1 and it is not involved in RPA loading at damaged chromatin. Thereby, we propose that LAP $2 \alpha / 2$ RE acts as a separation-of-function mutant that could be used to successfully differentiate LAP2 $\alpha$ governed biological process, and LAP2 $\alpha$-promoted RPA loading and genome integrity is, at least, uncoupled from pRB- or/and Lamin A/C-controlled signalling pathway. However, additional changes induced by LAP $2 \alpha$ deficiency cannot be overlooked, exemplified by the findings that LAP2 $\alpha$ is potentially involved in telomere maintenance [67] as well as 53BP1 accumulation [61]. The intrinsic ssDNA binding activity of RPA excludes the possibility that LAP2 $\alpha$ functions to directly recruit RPA, whereas it remains an open question that whether LAP2 $\alpha$ is involved in the controlling of RPA removal or retention, which may also affect RPA accumulation at damaged chromatin. It has been reported that LAP2 $\alpha$ likely behaves as a tumor suppressor through repressing the transcriptional activity of E2F by forming complex with $\mathrm{pRb}$ and Lamin A/C [34]. Given the essentiality of LAP2 $\alpha$-promoted RPA loading in genome stability, it will be worth exploring that whether LAP2 $\alpha$-promoted RPA loading or its dysregulation plays a role in tumorigenesis.

\section{Conclusions}

Our study provides mechanistic insight of RPA deposition in response to DNA damage including replication stress and DSBs. We propose that LAP2 $\alpha$ functions as a chaperone factor for RPA loading to preserve genome stability in mammalian cells. 


\section{Methods}

For a detailed description of all methods, see the Additional file 4: Supplemental Methods.

\section{Immunofluorescence}

Cells on glass coverslips (BD Biosciences) were fixed with 4\% paraformaldehyde and permeabilized with $0.2 \%$ Triton X-100 in PBS. Samples were blocked in 5\% donkey serum in the presence of $0.1 \%$ Triton X-100 and stained with the appropriate primary and secondary antibodies coupled to Alexa Fluor 488, 594, or 647 (Invitrogen). Confocal images were captured on a Zeiss LSM 900 microscope with a $\times 63$ oil objective. To avoid bleed-through effects in double-staining experiments, each dye was scanned independently in a multi-tracking mode. When inspection of nuclear wide dispersed RPA1, RPA2, or RPA2 pS33 foci, cells were pre-treated with $0.5 \%$ Triton X-100 for 5 min on ice to extract non-chromatin fractions and fixed with $3 \%$ paraformaldehyde and $2 \%$ sucrose for $15 \mathrm{~min}$ at room temperature. Cells were then permeabilized with $0.5 \%$ Triton X-100 for 5 min on ice then incubated in blocking buffer $(0.1 \%$ Triton $\mathrm{X}-100,5 \%$ donkey serum in $\mathrm{PBS}$ ) for $1 \mathrm{~h}$ at room temperature. For non-denaturing BrdU staining, cells were labelled with $10 \mu \mathrm{M}$ BrdU for $24 \mathrm{~h}$. For $\mathrm{S}$ phase discrimination, U2OS-LacO cells were pulsed with $10 \mu \mathrm{M}$ EdU at $37^{\circ} \mathrm{C}$ for $1 \mathrm{~h}$ before fixation. Incorporated EdU was click-labelled by using keyFluor 647-azide (Keygen Technologies) according to the manufacturer's instructions.

\section{Isolation of proteins on nascent DNA (iPOND)}

In brief, $2 \sim 3 \times 10^{8}$ cells were labelled with $10 \mu \mathrm{M}$ EdU (Thermo Fisher Scientific) for 10 min to detect nascent forks. For stalled forks, cells were treated with HU for $1 \mathrm{~h}$ in the continued presence of EdU. The harvested cells were then fixed with $1 \%$ formaldehyde in PBS solution for $20 \mathrm{~min}$ at room temperature followed by quenching of the crosslinking reaction with $1.25 \mathrm{M}$ glycine. Cells were then harvested and incubated in a permeabilization buffer $(0.25 \%$ Triton X-100/PBS) at room temperature for $30 \mathrm{~min}$. Then, cells were washed at $4{ }^{\circ} \mathrm{C}$ with $0.5 \% \mathrm{BSA} / \mathrm{PBS}$, then PBS alone, followed by incubation in the "click" (10 $\mathrm{mM}$ sodium ascorbate, $2 \mathrm{mM} \mathrm{CuSO}_{4}, 10 \mathrm{mM}$ biotin-azide, in PBS) or "no-click" (i.e., no biotin-azide) reaction cocktail for $2 \mathrm{~h}$ at room temperature. After the reaction, cells were re-suspended in lysis buffer (1\% SDS, $50 \mathrm{mM}$ Tris- $\mathrm{HCl}, \mathrm{pH}$ 8.0) containing protease inhibitor (Roche) and cell lysates were sonicated four times by using a Bioruptor (Diagenode) at $4{ }^{\circ} \mathrm{C}(30 \mathrm{~s}$ on and $30 \mathrm{~s}$ off) to generate $200-400 \mathrm{bp}$ DNA fragments. After centrifugation, EdU-labelled DNA was immunoprecipitated from supernatants by incubation with streptavidin-MyOne T1 beads (Thermo Fisher Scientific; pre-washed three times with PBS) for $4 \mathrm{~h}$ at $4{ }^{\circ} \mathrm{C}$. The streptavidin-agarose beads containing the captured DNA-protein complexes were then centrifuged for $3 \mathrm{~min}$ at $1800 \mathrm{~g}$. After washing for 5 min each with $1 \mathrm{~mL}$ cold lysis buffer, $1 \mathrm{~mL}$ of $1 \mathrm{M} \mathrm{NaCl}$, and twice more with $1 \mathrm{~mL}$ lysis buffer, the beads were supplemented 1:1 (v/v of packed beads) with $2 \times$ SDS-PAGE loading buffer and incubated at $95{ }^{\circ} \mathrm{C}$ for 25 min to liberate the proteins. SDS-PAGE fractionation and immunoblotting were then performed. 
ssDNA-pull down

A total of 30 pmol biotin-labelled 70-nt ssDNA (TGCAGCTGGCACGACAGGTTT TAATGAATCGGCCAACGCGCGGGGAGAGGCGGTTTGCGTATTGGGCGCT) and $30 \mu \mathrm{L}$ streptavidin Sepharose beads were preincubated in PBS with rotation at $4{ }^{\circ} \mathrm{C}$ overnight. Then, one third of the biotin-labelled ssDNA was incubated with nuclear extracts in NETN buffer at $4{ }^{\circ} \mathrm{C}$ for $8 \mathrm{~h}$ or at room temperature for $1 \mathrm{~h}$ followed by washing and immunoblotting. Oligonucleotide titrations were performed with 1-, 5-, and 20-fold molar excesses of dT10 or dT30. The ssDNA was added to the immobilized LAP2 $\alpha /$ RPA complexes and incubated for $1 \mathrm{~h}$ at room temperature under gentle rotation. After washing, bound RPA was analyzed by immunoblotting.

\section{DNA fiber}

To check fork symmetry, new origin firing, and fork speed, cells were first labelled with IdU $(25 \mu \mathrm{M})$ for 20 min, washed twice with media, and labelled with CldU (200 $\mu \mathrm{M})$ for $20 \mathrm{~min}$. To check fork degradation, cells were first labelled with IdU $(25 \mu \mathrm{M})$ for $20 \mathrm{~min}$, washed twice with media, and labelled with CldU $(200 \mu \mathrm{M})$ for $20 \mathrm{~min}$. After washing, cells were incubated with $4 \mathrm{mM} \mathrm{HU} \pm 100 \mu \mathrm{M}$ mirin for $4 \mathrm{~h}$. Cells were then trypsinized and re-suspended in PBS to $7 \times 10^{5} \mathrm{cells} / \mathrm{ml}$. Then, $2 \mu \mathrm{l}$ cells were mixed with $10 \mu \mathrm{l}$ lysis buffer (200 mM Tris-HCl, pH 7.4, $50 \mathrm{mM}$ EDTA, and 0.5\% SDS) on a clean glass slide. After 2 min incubation, the slides were tilted at $15^{\circ}$ to horizontal, allowing the lysate to slowly flow down along the slide. The slides were then air-dried, fixed in 3:1 methanol/acetic acid, and treated with $2.5 \mathrm{M} \mathrm{HCl}$ for $80 \mathrm{~min}$. The slides were then blocked (5\% BSA in PBS) for $30 \mathrm{~min}$ and incubated with antiBrdU antibodies (BD Bioscience, 347580 against IdU, and AbD Serotec, MCA2060GA against $\mathrm{CldU}$ ) in blocking buffer overnight. After washing, secondary antibodies coupled to Alexa Fluor 488 and 594 were diluted in PBS containing 5\% BSA and incubated with cells at room temperature for $1 \mathrm{~h}$. The slides were then washed 3 times with PBS. After washing, cells were mounted with an anti-fade solution and visualized under a Zeiss LSM 900 Fluorescence microscope. The length of all discrete fibers was measured by using ImageJ software. Fork symmetry was analyzed by measuring the length of CldU fiber (red) on each side. Fork speed was analyzed by measuring the length of CldU fiber based on an average fork elongation rate $(1 \mu \mathrm{m}$ roughly corresponds to $2 \mathrm{~kb}$ for each fiber). New origin firings were measured by counting only red fibers and compared to total fiber numbers.

\section{Statistics}

Data from biological triplicate or duplicate experiments are presented as mean \pm SDs. All statistical analyses involved were performed with SPSS 19. Two-tailed unpaired Student's $t$ test was used for comparing two groups of data. ANOVA with Bonferroni's correction was used to compare multiple groups of data. For values not normally distributed, Mann-Whitney $U$ test was used. $P<0.05$ was considered statistically significant. Before statistical analysis, variation within each group of data and the assumptions of the tests were checked. 


\section{Supplementary Information}

The online version contains supplementary material available at https://doi.org/10.1186/s13059-022-02638-6.

Additional file 1: Table S1. SILAC-based quantitative mass spectrometry analysis of RPA1-containing protein complexes.

Additional file 2: Figure S1. (Fig. 3 continued). LAP2a promotes the loading of RPA onto damaged chromatin. Figure S2. (Fig. 5 continued). LAP2a-promoted RPA loading is required for ATR activation and homologous recombination. Figure S3. (Fig. 6 continued). LAP2 is engaged into damaged chromatin in a PARP1-dependent manner.

Additional file 3: Table S2. Mass spectrometry analysis of LAP2a-containing protein complexes.

Additional file 4. Supplemental Methods.

Additional file 5. Uncropped versions of all blots.

Additional file 6. Review history.

\section{Acknowledgements}

We thank Dr. Xingzhi Xu and Dr. Bin Peng (Shenzhen University, Shenzhen, China) for providing help on laser micro-IR experiments. We thank Dr. Song Xiang, Dr. Hongquan Duan, and Dr. Hong Yang (Tianjin Medical University, Tianjin, China) for providing help on protein purification, EMSA and BRET assays. We thank Dr. Baohua Liu (Shenzhen University, Shenzhen, China) for providing $\mathrm{Lmna}^{+/+}$and $\mathrm{Lmna}^{-/-} \mathrm{MEFs}$.

\section{Review history}

The review history is available as Additional file 6.

\section{Peer review information}

Andrew Cosgrove was the primary editor of this article and managed its editorial process and peer review in collaboration with the rest of the editorial team.

\section{Authors' contributions}

KB, QZ, SL, NS, and LS conceived this project; KB, QZ, SL, NS, QG, LL, and ST conducted experiments; KB, QZ, SL, NS, QG, $\mathrm{LL}, \mathrm{ST}$, and $K Z$ acquired data; KB, QZ, SL, NS, QG, LL, ST, KZ, and LS analyzed data; JH, YZ, KZ, DA, JY, ZY, and RF provided technical supports and reagents; KB, QZ, SL, NS, and LS wrote the manuscript. All author(s) read and approved the final manuscript.

\section{Funding}

This work was supported by grant (2019YFA0802003 to Y.Z.) from Chinese Ministry of Science and Technology, grants (81972660 and 81722036 to L.S., 81902696 to N.S., and 81502408 to L.L.) from the National Natural Science Foundation of China, grants (17JCJQJC46100 to L.S. and 17JCJQJC45700 to D.A.) from Tianjin Funds for Distinguished Young Scientists, and grant (18JCZDJC97400 to L. S.) from National Natural Science Foundation of Tianjin.

\section{Availability of data and materials}

All data generated or analyzed during this study are included in this published article and its supplementary information files. The mass spectrometry proteomics data have been deposited to the ProteomeXchange Consortium (http:// proteomecentral.proteomexchange.org) via the iProX partner repository [68] with the dataset identifier PXD028105. All microscopy images are available at Figshare and can be accessed at (high content screening replicate 1: doi.org/10.6084/ m9.figshare.19070321 [69], high content screening replicate 2: doi.org/10.6084/m9.figshare.19074440 [69], and other images: doi.org/10.6084/m9.figshare.c.5814131) [70].

\section{Declarations}

\section{Ethics approval and consent to participate}

All procedures involving animals were approved by the Ethics Committee of the Tianjin Medical University with reference No. TMUaMEC 2021035 and followed the NIH Guide for the Care and Use of Laboratory Animals (8th ed. The National Academies Press, 2011).

\section{Consent for publication}

Not applicable.

\section{Competing interests}

The authors declare that they have no competing interests.

\section{Author details}

${ }^{1}$ State Key Laboratory of Experimental Hematology, The Province and Ministry Co-sponsored Collaborative Innovation Center for Medical Epigenetics, Key Laboratory of Immune Microenvironment and Disease (Ministry of Education), Key Laboratory of Breast Cancer Prevention and Therapy (Ministry of Education), Tianjin Medical University Cancer Institute and Hospital, Tianjin Medical University General Hospital, School of Basic Medical Sciences, Tianjin Medical University, Tianjin 300070, China. ${ }^{2}$ Department of Clinical Laboratory, First Affiliated Hospital of Xi'an Jiaotong University, Xi'an 710061, Shaanxi, China. ${ }^{3}$ Max Perutz Laboratories, Center of Medical Biochemistry, Medical University of Vienna, Vienna Biocenter (VBC), Vienna, Austria. ${ }^{4}$ Department of Biochemistry and Molecular Biology, Tianjin Medical University, 22 Qixiangtai Road, Tianjin 300070, China. 
Received: 3 June 2021 Accepted: 17 February 2022

Published online: 28 February 2022

\section{References}

1. Buisson R, Boisvert $J$, Benes CH, Zou L. Distinct but concerted roles of ATR, DNA-PK, and Chk1 in countering replication stress during $S$ phase. Mol Cell. 2015;59:1011-24.

2. Cimprich KA, Cortez D. ATR: an essential regulator of genome integrity. Nat Rev Mol Cell Biol. 2008;9:616-27.

3. Blackford AN, Jackson SP. ATM, ATR, and DNA-PK: the trinity at the heart of the DNA damage response. Mol Cell. 2017;66:801-17.

4. Halazonetis TD, Gorgoulis VG, Bartek J. An oncogene-induced DNA damage model for cancer development. Science. 2008;319:1352-5.

5. Bartkova J, Horejsi Z, Koed K, Kramer A, Tort F, Zieger K, et al. DNA damage response as a candidate anti-cancer barrier in early human tumorigenesis. Nature. 2005;434:864-70.

6. Gorgoulis VG, Vassiliou LV, Karakaidos P, Zacharatos P, Kotsinas A, Liloglou T, et al. Activation of the DNA damage checkpoint and genomic instability in human precancerous lesions. Nature. 2005;434:907-13.

7. Saldivar JC, Cortez D, Cimprich KA. The essential kinase ATR: ensuring faithful duplication of a challenging genome. Nat Rev Mol Cell Biol. 2017;18:622-36.

8. Lans H, Hoeijmakers JHJ, Vermeulen W, Marteijn JA. The DNA damage response to transcription stress. Nat Rev Mol Cell Biol. 2019;20:766-84

9. Matos DA, Zhang JM, Ouyang J, Nguyen HD, Genois MM, Zou L. ATR protects the genome against R loops through a MUS81-triggered feedback loop. Mol Cell. 2019;77:514-27.e4.

10. Quinet A, Lemacon D, Vindigni A. Replication fork reversal: players and guardians. Mol Cell. 2017;68:830-3.

11. Zou L, Elledge SJ. Sensing DNA damage through ATRIP recognition of RPA-ssDNA complexes. Science. 2003;300:1542-8

12. Toledo LI, Altmeyer M, Rask MB, Lukas C, Larsen DH, Povlsen LK, et al. ATR prohibits replication catastrophe by preventing global exhaustion of RPA. Cell. 2013;155:1088-103.

13. Marechal A, Zou L. RPA-coated single-stranded DNA as a platform for post-translational modifications in the DNA damage response. Cell Res. 2015;25:9-23.

14. Friedel AM, Pike BL, Gasser SM. ATR/Mec1: coordinating fork stability and repair. Curr Opin Cell Biol. 2009;21:237-44.

15. Dungrawala H, Rose KL, Bhat KP, Mohni KN, Glick GG, Couch FB, et al. The replication checkpoint prevents two types of fork collapse without regulating replisome stability. Mol Cell. 2015;59:998-1010.

16. Wang Y, Putnam CD, Kane MF, Zhang W, Edelmann L, Russell R, et al. Mutation in Rpa1 results in defective DNA double-strand break repair, chromosomal instability and cancer in mice. Nat Genet. 2005;37:750-5.

17. Wang G, Li Y, Wang P, Liang H, Cui M, Zhu M, et al. PTEN regulates RPA1 and protects DNA replication forks. Cell Res. 2015;25:1189-204.

18. Fang Y, Tsao CC, Goodman BK, Furumai R, Tirado CA, Abraham RT, et al. ATR functions as a gene dosage-dependent tumor suppressor on a mismatch repair-deficient background. EMBO J. 2004;23:3164-74.

19. Brown EJ, Baltimore D. ATR disruption leads to chromosomal fragmentation and early embryonic lethality. Genes Dev. 2000;14:397-402.

20. Gilad O, Nabet BY, Ragland RL, Schoppy DW, Smith KD, Durham AC, et al. Combining ATR suppression with oncogenic Ras synergistically increases genomic instability, causing synthetic lethality or tumorigenesis in a dosage-dependent manner. Cancer Res. 2010;70:9693-702.

21. Murga M, Bunting S, Montana MF, Soria R, Mulero F, Canamero M, et al. A mouse model of ATR-Seckel shows embryonic replicative stress and accelerated aging. Nat Genet. 2009;41:891-8.

22. Bonilla B, Hengel SR, Grundy MK, Bernstein KA. RAD51 gene family structure and function. Annu Rev Genet. 2020;54:25-46.

23. Wold MS. Replication protein a: a heterotrimeric, single-stranded DNA-binding protein required for eukaryotic DNA metabolism. Annu Rev Biochem. 1997;66:61-92.

24. Bochkareva E, Korolev S, Lees-Miller SP, Bochkarev A. Structure of the RPA trimerization core and its role in the multistep DNA-binding mechanism of RPA. EMBO J. 2002;21:1855-63.

25. Fan J, Pavletich NP. Structure and conformational change of a replication protein a heterotrimer bound to ssDNA. Genes Dev. 2012;26:2337-47.

26. Chen $\mathrm{R}$, Subramanyam S, Elcock AH, Spies M, Wold MS. Dynamic binding of replication protein a is required for DNA repair. Nucleic Acids Res. 2016;44:5758-72.

27. Salas TR, Petruseva I, Lavrik O, Saintome C. Evidence for direct contact between the RPA3 subunit of the human replication protein a and single-stranded DNA. Nucleic Acids Res. 2009;37:38-46.

28. Brosey CA, Chagot ME, Ehrhardt M, Pretto DI, Weiner BE, Chazin WJ. NMR analysis of the architecture and functional remodeling of a modular multidomain protein, RPA. J Am Chem Soc. 2009;131:6346-7.

29. Brosey CA, Yan C, Tsutakawa SE, Heller WT, Rambo RP, Tainer JA, et al. A new structural framework for integrating replication protein a into DNA processing machinery. Nucleic Acids Res. 2013;41:2313-27.

30. Yang Q, Zhu Q, Lu X, Du Y, Cao L, Shen C, et al. G9a coordinates with the RPA complex to promote DNA damage repair and cell survival. Proc Natl Acad Sci U S A. 2017;114:E6054-63.

31. Gong Z, Kim JE, Leung CC, Glover JN, Chen J. BACH1/FANCJ acts with TopBP1 and participates early in DNA replication checkpoint control. Mol Cell. 2010;37:438-46.

32. Szambowska A, Tessmer I, Prus P, Schlott B, Pospiech H, Grosse F. Cdc45-induced loading of human RPA onto single-stranded DNA. Nucleic Acids Res. 2017:45:3217-30. 
33. Li S, Xu Z, Xu J, Zuo L, Yu C, Zheng P, et al. Rtt105 functions as a chaperone for replication protein a to preserve genome stability. EMBO J. 2018;37:e99154.

34. Brachner A, Foisner R. Lamina-associated polypeptide (LAP)2alpha and other LEM proteins in cancer biology. Adv Exp Med Biol. 2014;773:143-63.

35. Mirza AN, McKellar SA, Urman NM, Brown AS, Hollmig T, Aasi SZ, et al. LAP2 proteins chaperone GLI1 movement between the lamina and chromatin to regulate transcription. Cell. 2019;176:198-212 e115.

36. Bradley CM, Jones S, Huang Y, Suzuki Y, Kvaratskhelia M, Hickman AB, et al. Structural basis for dimerization of LAP2alpha, a component of the nuclear lamina. Structure. 2007;15:643-53.

37. Naetar N, Korbei B, Kozlov S, Kerenyi MA, Dorner D, Kral R, et al. Loss of nucleoplasmic LAP2alpha-Lamin a complexes causes erythroid and epidermal progenitor hyperproliferation. Nat Cell Biol. 2008;1 0:1341-8.

38. Petermann E, Orta ML, Issaeva N, Schultz N, Helleday T. Hydroxyurea-stalled replication forks become progressively inactivated and require two different RAD51-mediated pathways for restart and repair. Mol Cell. 2010;37:492-502.

39. Shiotani B, Nguyen HD, Hakansson P, Marechal A, Tse A, Tahara H, et al. Two distinct modes of ATR activation orchestrated by Rad17 and Nbs1. Cell Rep. 2013;3:1651-62.

40. Ma S, Cao C, Che S, Wang Y, Su D, Liu S, et al. PHF8-promoted TOPBP1 demethylation drives ATR activation and preserves genome stability. Sci Adv. 2021;7:eabf7684.

41. Ray Chaudhuri A, Hashimoto Y, Herrador R, Neelsen KJ, Fachinetti D, Bermejo R, et al. Topoisomerase I poisoning results in PARP-mediated replication fork reversal. Nat Struct Mol Biol. 2012;19:417-23.

42. Vujanovic M, Krietsch J, Raso MC, Terraneo N, Zellweger R, Schmid JA, et al. Replication fork slowing and reversal upon DNA damage require PCNA polyubiquitination and ZRANB3 DNA translocase activity. Mol Cell. 2017:67:882-90 e885.

43. Tang J, Cho NW, Cui G, Manion EM, Shanbhag NM, Botuyan MV, et al. Acetylation limits 53BP1 association with damaged chromatin to promote homologous recombination. Nat Struct Mol Biol. 2013;20:317-25.

44. Kim J, Sturgill D, Sebastian R, Khurana S, Tran AD, Edwards GB, et al. Replication stress shapes a protective chromatin environment across fragile genomic regions. Mol Cell. 2018;69:36-47 e37.

45. Redwood AB, Perkins SM, Vanderwaal RP, Feng Z, Biehl KJ, Gonzalez-Suarez I, et al. A dual role for A-type lamins in DNA double-strand break repair. Cell Cycle. 2011;10:2549-60.

46. Gibbs-Seymour I, Markiewicz E, Bekker-Jensen S, Mailand N, Hutchison CJ. Lamin a/C-dependent interaction with 53BP1 promotes cellular responses to DNA damage. Aging Cell. 2015;14:162-9.

47. Li W, Bai X, Li J, Zhao Y, Liu J, Zhao H, et al. The nucleoskeleton protein IFFO1 immobilizes broken DNA and suppresses chromosome translocation during tumorigenesis. Nat Cell Biol. 2019;21:1273-85.

48. Bhat KP, Cortez D. RPA and RAD51: fork reversal, fork protection, and genome stability. Nat Struct Mol Biol. 2018;25:446-53.

49. Couch FB, Bansbach CE, Driscoll R, Luzwick JW, Glick GG, Betous R, et al. ATR phosphorylates SMARCAL1 to prevent replication fork collapse. Genes Dev. 2013;27:1610-23.

50. Wang Q, Ma S, Song N, Li X, Liu L, Yang S, et al. Stabilization of histone demethylase PHF8 by USP7 promotes breast carcinogenesis. J Clin Invest. 2016;126:2205-20.

51. Su D, Ma S, Shan L, Wang Y, Wang Y, Cao C, et al. Ubiquitin-specific protease 7 sustains DNA damage response and promotes cervical carcinogenesis. J Clin Invest. 2018;128:4280-96.

52. Yazinski SA, Comaills V, Buisson R, Genois MM, Nguyen HD, Ho CK, et al. ATR inhibition disrupts rewired homologous recombination and fork protection pathways in PARP inhibitor-resistant BRCA-deficient cancer cells. Genes Dev. 2017;31:318-32.

53. Kim H, George E, Ragland R, Rafail S, Zhang R, Krepler C, et al. Targeting the ATR/CHK1 axis with PARP inhibition results in tumor regression in BRCA-mutant ovarian cancer models. Clin Cancer Res. 2017;23:3097-108.

54. Lord CJ, Ashworth A. PARP inhibitors: synthetic lethality in the clinic. Science. 2017:355:1152-8.

55. Ahel D, Horejsi Z, Wiechens N, Polo SE, Garcia-Wilson E, Ahel I, et al. Poly(ADP-ribose)-dependent regulation of DNA repair by the chromatin remodeling enzyme ALC1. Science. 2009;325:1240-3.

56. Mortusewicz O, Ame JC, Schreiber V, Leonhardt H. Feedback-regulated poly(ADP-ribosyl)ation by PARP-1 is required for rapid response to DNA damage in living cells. Nucleic Acids Res. 2007;35:7665-75.

57. Mimitou EP, Symington LS. DNA end resection: many nucleases make light work. DNA Repair (Amst). 2009;8:983-95

58. Nishino T, Morikawa K. Structure and function of nucleases in DNA repair: shape, grip and blade of the DNA scissors. Oncogene. 2002;21:9022-32.

59. Jungmichel S, Rosenthal F, Altmeyer M, Lukas J, Hottiger MO, Nielsen ML. Proteome-wide identification of poly(ADP-Ribosyl)ation targets in different genotoxic stress responses. Mol Cell. 2013;52:272-85.

60. Gagne JP, Pic E, Isabelle M, Krietsch J, Ethier C, Paquet E, et al. Quantitative proteomics profiling of the poly(ADPribose)-related response to genotoxic stress. Nucleic Acids Res. 2012;40:7788-805.

61. Bartova E, Legartova S, Krejci J, Reznickova P, Kovarikova AS, Suchankova J, et al. Depletion of A-type lamins and Lap2alpha reduces 53BP1 accumulation at UV-induced DNA lesions and Lap2alpha protein is responsible for compactness of irradiated chromatin. J Cell Biochem. 2018;119:8146-62.

62. Gesson K, Rescheneder P, Skoruppa MP, von Haeseler A, Dechat T, Foisner R. A-type lamins bind both hetero- and euchromatin, the latter being regulated by lamina-associated polypeptide 2 alpha. Genome Res. 2016;26:462-73.

63. Vidak S, Kubben N, Dechat T, Foisner R. Proliferation of progeria cells is enhanced by lamina-associated polypeptide 2alpha (LAP2alpha) through expression of extracellular matrix proteins. Genes Dev. 2015:29:2022-36.

64. Dorner D, VIcek S, Foeger N, Gajewski A, Makolm C, Gotzmann J, et al. Lamina-associated polypeptide 2alpha regulates cell cycle progression and differentiation via the retinoblastoma-E2F pathway. J Cell Biol. 2006;173:83-93.

65. Dechat T, Korbei B, Vaughan OA, Vlcek S, Hutchison CJ, Foisner R. Lamina-associated polypeptide 2alpha binds intranuclear A-type lamins. J Cell Sci. 2000;113(Pt 19):3473-84. 
66. Shumaker DK, Lee KK, Tanhehco YC, Craigie R, Wilson KL. LAP2 binds to BAF.DNA complexes: requirement for the LEM domain and modulation by variable regions. EMBO J. 2001;20:1754-64.

67. Dechat T, Gajewski A, Korbei B, Gerlich D, Daigle N, Haraguchi T, et al. LAP2alpha and BAF transiently localize to telomeres and specific regions on chromatin during nuclear assembly. J Cell Sci. 2004;117:6117-28.

68. Ma J, Chen T, Wu S, Yang C, Bai M, Shu K, et al. iProX: an integrated proteome resource. Nucleic Acids Res. 2019;47:D1211-7.

69. Bao K, Zhang Q, Liu S, Song N, Guo Q, Liu L, et al. High content imaging analysis of foci intensity of RPA1 and RPA2 in U2OS cells. 2022. https://doi.org/10.6084/m9.figshare.19070321.

70. Bao K, Zhang Q, Liu S, Song N, Guo Q, Liu L, et al. LAP2 a preserves genome integrity through assisting RPA deposition on damaged chromatin. 2022. https://doi.org/10.6084/m9.figshare.c.5814131.

\section{Publisher's Note}

Springer Nature remains neutral with regard to jurisdictional claims in published maps and institutional affiliations.

- fast, convenient online submission

- thorough peer review by experienced researchers in your field

- rapid publication on acceptance

- support for research data, including large and complex data types

- gold Open Access which fosters wider collaboration and increased citations

- maximum visibility for your research: over 100M website views per year

At BMC, research is always in progress.

Learn more biomedcentral.com/submissions 